\title{
Reappraisal of thyroxine treatment in primary hypothyroidism
}

\author{
S Hodges, B P O'Malley, B N Northover, K L Woods, P G F Swift
}

\begin{abstract}
The optimum daily dose of thyroxine was calculated for 13 children aged 3-16 years with primary hypothyroidism by titrating their doses at monthly intervals. The condition of the thyroid was assessed by sensitive assay of thyroid stimulating hormone concentrations, as well as measurement of total and free thyroid hormone concentrations and systolic time interval ratios. Serum thyroid stimulating hormone concentration was found to be the most responsive to small changes in thyroxine. The calculated optimum daily replacement dose of thyroxine $\left(102 \mu \mathrm{g} / \mathrm{m}^{2}\right.$ or $\left.3.5 \mu \mathrm{g} / \mathrm{kg}\right)$ was fractionally lower than that previously recommended, and was more closely related to surface area (coefficient of variation $8 \cdot 2 \%$ ) than to body weight (coefficient of variation $16.2 \%)$.

Our results suggest that though monthly may be the optimal time interval for increases in the dose of thyroxine, any reduction in the dose should be made more gradually.
\end{abstract}

Neonatal screening for congenital hypothyroidism together with adequate treatment should allow normal physical and intellectual development. For optimal results careful adjustment of the dose of thyroxine according to age and body size is necessary. An inadequate dose may affect intellectual and neurological development adversely, ${ }^{1}$ whereas excessive replacement may lead to craniostenosis, ${ }^{2}{ }^{3}$ advanced bone age, and hyperactivity. ${ }^{4}$ In 1979 Rezvani and Di George suggested a daily dose of $105 \mu \mathrm{g} / \mathrm{m}^{2}$, or $3.8 \mu \mathrm{g} / \mathrm{kg}$ for hypothyroid children aged more than 1 year; this was significantly less than the previously recommended doses. Several subsequent developments, paticularly the $11 \%$ increase in bioavailable thyroxine content of the British Pharmaceutical Codex formulation (British Pharmacopoeia 1980), the introduction of $25 \mu \mathrm{g}$ tablets in the same year, and the advent of sensitive immunoradiometric assays (IRMA) for the measurement of serum thyroid stimulating hormone concentrations have justified a reappraisal of the optimal doses of thyroxine for hypothyroid children.

After the introduction of the highly sensitive thyroid stimulating hormone IRMA we became concerned that a number of children in the clinic had unmeasurably low concentrations, and thus could be considered to be over treated. The aim of this study, therefore, was to determine the 'optimal' dose of thyroxine, defining this as the minimal dose at which the thyroid stimu-

lating hormone was suppressed sufficiently to come within the reference range. We have also tried to determine whether the systolic time interval ratio (pre-ejection period:left ventricular ejection time), which has been established as a reliable measure of end organ response to thyroid hormones in adults, ${ }^{67}$ could be used as a physiological marker of adequacy of replacement in children.

\section{Patients and methods}

Fifteen patients with previously diagnosed primary hypothyroidism were investigated. Informed parental consent was obtained and the study was approved by the local ethics committee.

Thirteen children (nine girls and four boys) aged between 3 and 16 years (mean age 10.3) completed the study. Seven had congenital hypothyroidism and six had juvenile hypothyroidism. Nine of the 13 children were prepubertal. The children were seen at monthly intervals. At the initial visit the weight, height, and dose of thyroxine were recorded and bone age was estimated by the method of Greulich and Pyle. ${ }^{8} \mathrm{~A}$ blood sample was taken by venepuncture and the serum was divided into two aliquots. In one aliquot thyroid stimulating hormone (Boots Celltech IRMA) was estimated with seven days as a guide to dose adjustment. The second was stored at $-20^{\circ} \mathrm{C}$ until the end of the study when all the serum samples of each patient were assayed for free triiodothyroxine $\left(\mathrm{T}_{3}\right)$ (Immunodiagnostics Ltd) (reference range 3.6-7.8 pmol/l), free thyroxine $\left(\mathrm{T}_{4}\right)$ (Clinical Assays 2 stage, Baxter Health Care) (reference range $7 \cdot 7-20.6 \mathrm{pmol} / \mathrm{l}$ ), and thyroid stimulating hormone again, in their respective single assay runs. If the initial thyroid stimulating hormone concentration was within the reference range $(0.3-4 \mathrm{mU} / \mathrm{l})(\mathrm{n}=12)$ the dose of thyroxine was reduced by $12.5 \mu \mathrm{g}$ at monthly intervals until the thyroid stimulating hormone concentration rose above $4 \mathrm{mU} / 1$. The dose was then increased by $12.5 \mu \mathrm{g}$ at monthly intervals until the thyroid stimulating hormone concentration was again within the reference range.

If the serum thyroid stimulating hormone concentration was initially raised $(>4 \mathrm{mU} / \mathrm{l})$ $(n=1)$ the dose of thyroxine was increased at monthly intervals until it returned to the reference range.

Data are presented for four time points: point 1 , entry to the study; point 2 , the last reduction in dose that kept the thyroid stimulating hormone concentration within the reference range; point 3 , the dose at which the thyroid stimulating hormone concentration rose above the refer-
Clinical Sciences Building, Leicester Royal Infirmary, Leicester LE2 7LX. Accepted 23 May 1990

Department of
Child Health,
University of Leicester
S Hodges
Department of
Pharmacology and
Therapeutics,
University of Leicester
B P O'Malley
K L Woods
Department of
Pharmacology,
Leicester Polytechnic
B N Northover
Department of
Paediatrics,
Leicester General
Hospital
P G F Swift
Correspondence to:
Dr B P O'Malley,
Department of Pharmacology
and Therapeutics,
Clinical Sciences Building,
Leicester Royal Infirmary,
Leicester LE2 7LX.
Accepted 23 May 1990


ence range; and point 4 , the point during the incremental dose adjustment at which the thyroid stimulating hormone concentration returned to the reference range.

At each clinic attendance pre-ejection period and left ventricular ejection times were measured according to a previously reported technique, ${ }^{9}$ and the systolic time interval ratio was calculated. The systolic time intervals were also calculated for 43 euthyroid children between the ages of 3 and 16 years to provide a reference range for the age group under study.

Patients were asked about symptoms suggestive of over replacement and under replacement at each attendance. Thyroxine was dispensed by the hospital pharmacy as $25 \mu \mathrm{g}$ or $50 \mu \mathrm{g}$ tablets and these were specially packed in accordance with the monthly titration requirements of the study. Detailed instructions were given to the parents. Tablets were counted at the beginning and end of each study period to assess compliance.

The significance of differences were assessed by the paired $t$ test for all data except thyroid stimulating hormone concentrations. These were no normally distributed and therefore the Wilcoxon test was used.

\section{Results}

The association between the thyroxine dose given $\left(\mu \mathrm{g} / \mathrm{m}^{2} /\right.$ day) and the thyroid stimulating hormone concentration at each time point is shown in fig 1 . At entry (time point 1 ) thyroid stimulating hormone was in the lower part of the reference range for the group as a whole, but moved higher during downward titration of the dose of thyroxine (time point 2). Further downward titration (until the thyroid stimulating hormone for each individual patient rose above the reference range point 3 ) was followed by upward titration to suppress the thyroid stimulting hormone of each patient until it was within the reference range (point 4). The thyroxine dose at point $2\left(91 \cdot 5 \mu \mathrm{g} / \mathrm{m}^{2}\right)$ was significantly lower than at point $4\left(102 \cdot 2 \mu \mathrm{g} / \mathrm{m}^{2}\right.$, $\mathrm{p}<0.005)$ despite the similarity in thyroid stimulating hormone concentrations at these

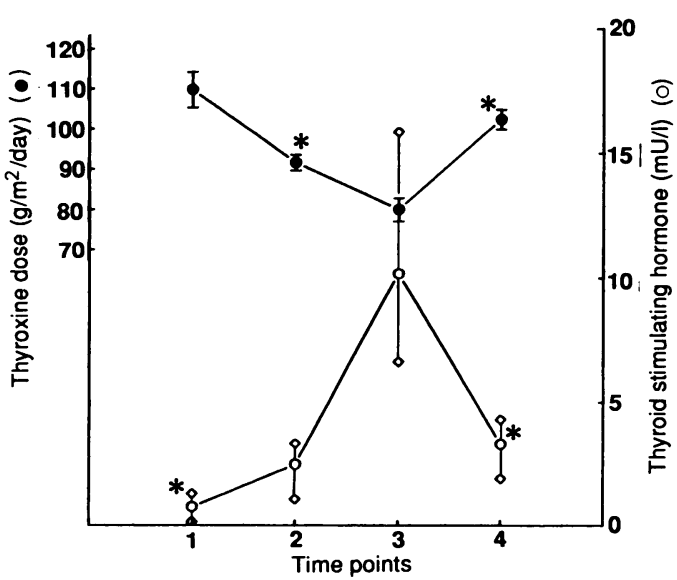

Figure 1 Thyroxine dose ( $\mu \mathrm{g} / \mathrm{m}^{2} /$ day) and concentration of thyroid stimulating hormone $(\mathrm{mU} / \mathrm{l})$ at each of the four time points of the study. $\left({ }^{*} p<0.005\right)$. Dose of thyroxine is expressed as mean (SEM) and concentration of thyroid stimulating hormone as median (interquartile range).

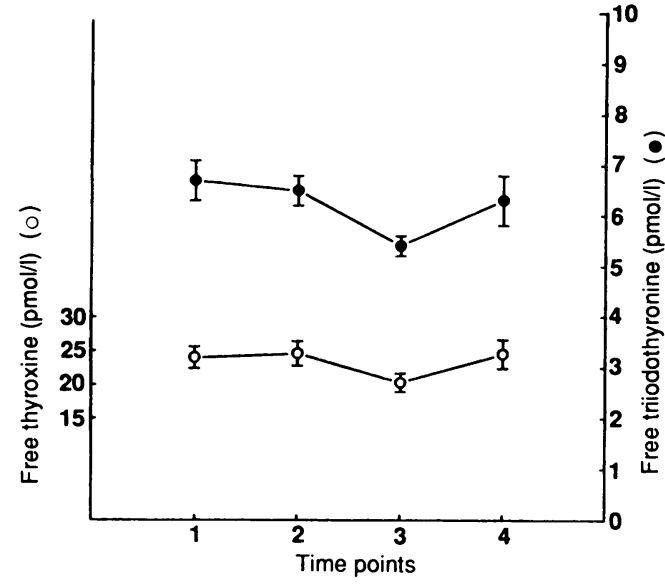

Figure 2 Free triiodothyronine (solid circles) and free thyroxine (open circles) concentrations at the four time points expressed as mean (SEM).

two points. Figure 2 shows that there was no significant difference at points 2 and 4 either in free triiodothyronine $(6 \cdot 4$ compared with $6 \cdot 3$ $\mathrm{pmol} / \mathrm{l}, \mathrm{p}=0 \cdot 34)$ or free thyroxine $(24 \cdot 6 \mathrm{com}$ pared with $24.5 \mathrm{pmol} / \mathrm{l}, \mathrm{p}=0.99$ ).

The final dose of thyroxine reached by upward titration until the thyroid stimulating hormone came within the reference range (point 4) was not significantly different from that at entry $\left(102 \cdot 2 \mu \mathrm{g} / \mathrm{m}^{2}\right.$ compared with $109 \cdot 6 \mu \mathrm{g} / \mathrm{m}^{2}$, $p=0 \cdot 16)$. The thyroid stimulating hormone concentration was, however, significantly lower at point 1 than at point $4(0.74$ compared with $3.30 \mathrm{mU} / 1, \mathrm{p}<0.005$ ), although neither free triiodothyronine nor free thyroxine concentrations differed significantly at points 1 and 4 $(p=0.45$ and 0.83 , respectively).

Figure 3 shows the measurements of systolic time interval of the study group at each time point in relation to the $80 \%$ prediction band calculated using the results from the group of 43 euthyroid children. One measurement is missing from time points $2-4$. The observed systolic time intervals at time points 1 and 4 were indistinguishable from those of the euthyroid population. At both time points 2 and 3, however, in a third of the study group the systolic time intervals were clearly outside the $80 \%$ prediction band, and two older children had unequivocally hypothyroid values.

The precision of estimating the thyroxine dose by body weight or surface area was compared by calculating coefficients of variation for these two measures at thyroid stimulating hormone euthyroidism (points 2 and 4 pooled). The coefficient of variation was $8 \cdot 2 \%$ for the dose expressed by $\mathrm{m}^{2}$ and $16 \cdot 2 \%$ when expressed by $\mathrm{kg}$ body weight. The regression of dose by surface area at time point 4 is shown in fig 4.

When juvenile and congenital hypothyroid patients were considered separately, results did not differ appreciably.

At entry to the study two patients had symptoms suggestive of hyperthyroidism (over activity and poor sleeping). Two girls were withdrawn; one with Hashimoto's thyroiditis complained of pain and swelling of her thyroid 


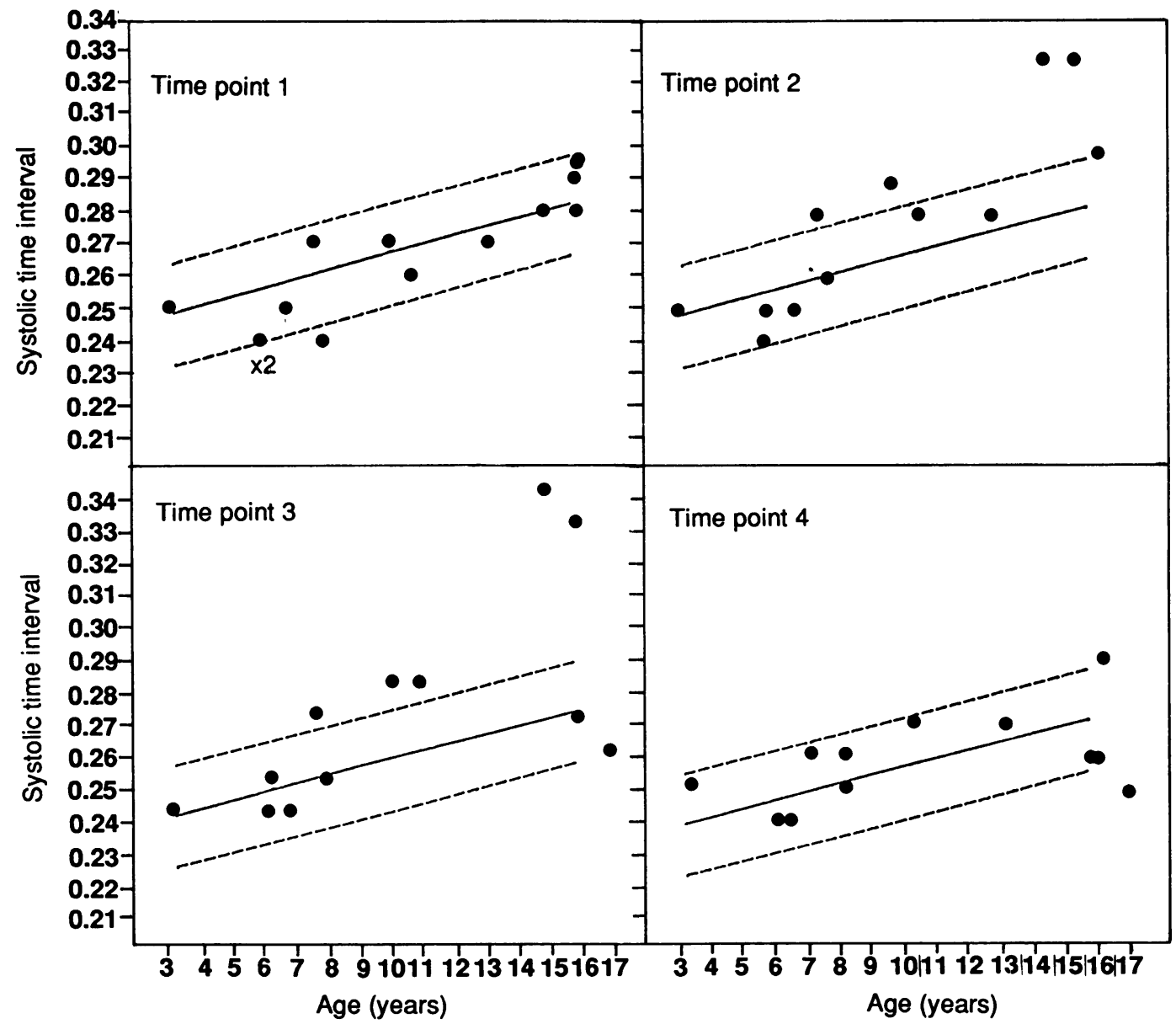

Figure 3 Systolic time intervals for each patient at the four time points. The regression line of systolic time interval on age (solid line) and its $80 \%$ prediction band (interrupted lines) for euthyroid children are shown.

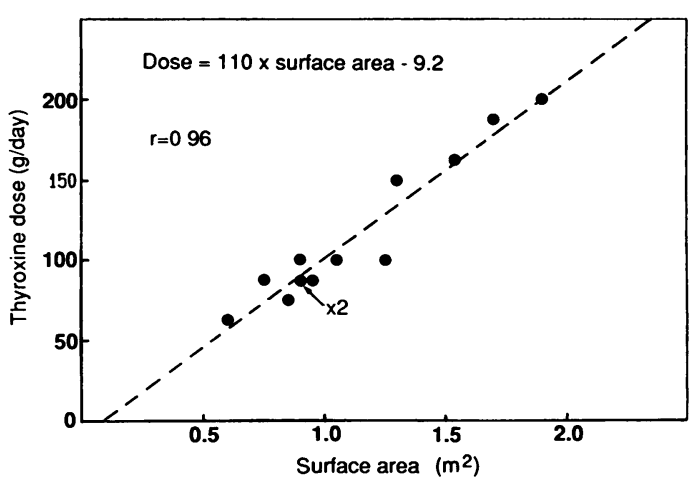

Figure 4 The relation between thyroxine dose ( $\mu \mathrm{g} /$ day) and body surface area (metre $)^{2}$ ) at time point 4 with the calculated regression equation and correlation coefficient.

gland when her dose of thyroxine was reduced although she remained biochemically euthyroid, and the other remained clinically and biochemically euthyroid after she had stopped taking thyroxine. At the end of the study all patients were free of symptoms and six of the study group were receiving smaller doses of thyroxine than they had been at entry.

\section{Discussion}

We have shown that in children with primary hypothyroidism the optimal daily dose of thyroxine defined as the minimum dose that suppresses the serum thyroid stimulating hormone until it is with the reference range, and determined with $12.5 \mu \mathrm{g}$ increments of thyroxine, is $102 \mu \mathrm{g} / \mathrm{m}^{2}$ (or $3.5 \mu \mathrm{g} / \mathrm{kg}$ ). This is fractionally lower than that previously recommended. Dosage requirements correlated better with surface area than body weight. Judging by measurements of systolic time interval, the children's cardiac tissue did not show evidence of over treatment at the time of inclusion in the study when compared with age matched controls in spite of their free thyroid hormone concentrations being at the upper limit of the adult reference range. Considering the group as a whole there were no consistent alterations in systolic time interval during titration of the dose of thyroxine, although about a third of the subjects developed hypothyroid values at the time of the raised serum thryroid stimulating hormone concentrations. The design of the study, in which doses were titrated downwards as well as upwards, has highlighted an interesting discrepancy. Lower optimal doses were arrived at during downward compared with upward titration even though thyroxine and triiodothyronine concentrations were similar at the respective optimal end points. This might be explained by the fact that a 28 day titration period is too short for adequate dose reduction. The serum half life of thyroxine is roughly six days ${ }^{10}$ and, if five times the half life is required to reach a steady state plasma concentration, 
there may still be some contribution to plasma concentrations from the immediately previous higher dose of thyroxine.

In contrast, a monthly interval between increments is sufficient to produce maximal thyroid stimulating hormone suppression during upward titration in adults. ${ }^{11}$ Sato et al showed that children with congenital hypothyroidism who, were studied between 6 months and 17 years of age required unusually large doses of thyroxine/kg body weight to block their thyroid stimulating hormone response to thyrotrophin releasing hormone. ${ }^{12}$ More recently, Schultz et al described five infants with congenital hypothyroidism who had persistently raised serum thyroid stimulating hormone concentrations after being given thyroxine in doses that would usually be considered adequate. ${ }^{13}$ These observations suggest that congenitally hypothyroid children have a particularly high threshold for thyroid stimulating hormone suppression by thyroxine. It could be argued that in our study the attainment of over suppressed thyroid stimulating hormone concentrations (as shown by the lower concentrations on admission to the study) after long term administration of similar doses of thyroxine to those which, during the study, lowered thyroid stimulating hormone just into the reference range supports the hypothesis of an initial high threshold for thyroid stimulating hormone suppression. Moreover, the pronounced rise in thyroid stimulating hormone seen in our subjects after a $12.5 \mu \mathrm{g}$ decrement of thyroxine (fig 1 ) raises the possibility that this increased threshold is the consequence of the driving effect of thyrotrophin releasing hormone on thyroid stimulating hormone release.

The discrepancy in dose requirements that we have identified is of some importance when reviewing previous reports. For instance, Rezvani and Di George suggested an optimal dose of $105 \mu \mathrm{g} / \mathrm{m}^{2}$ during downward titration, but this is likely to have been an under estimate of the dose required to produce thyroid stimulating hormone suppression during upward titration should they have adopted such. ${ }^{5}$

In adults the systolic time interval ratio is a reliable monitor of peripheral thyroid hormone activity. ${ }^{67}$ The systolic time intervals obtained in this study at the time of entry did not suggest that the children were over treated. It is likely that the variability of the systolic time interval with age (as observed in the control group) and the problems of achieving a resting state in young children limit the clinical usefulness of the systolic time interval ratio as an index of peripheral thyroid hormone activity in children.

We conclude that, in children with primary hypothyroidism, the optimal daily dose of thyroxine when prescribed in the current British Pharmaceutical Codex formulation, is slightly lower than previously recommended. The required dose correlates more closely with surface area than with body weight. Assay of thyroid stimulating hormone by IRMA provides a sensitive means of monitoring even minor dose adjustments, whereas the 'tissue marker', the systolic time interval, is less sensitive. The discrepancies between incremental and decremental titration may be of clinical relevance in that though the time interval of one month may be adequate between increments, it may be necessary to decrease thyroxine more gradually in children with over replacement if under replacement is to be avoided.

We thank the pharmacy department, Leicester Royal Infirmary, for packaging and dispensing the thyroxine.

1 Dussault JH, Letarte J, Glorieux J, Morissette J, Guyda H. Psychological development of hypothyroid infants at age 12 and 18 months: experience after neonatal screening. In: Burrow GN, Dussault JH, eds. Neonatal thyroid screening. New York: Raven Press, 1988:271-6.

2 Penfold JL, Simpson DA. Premature craniosynostosis - a complication of thyroid replacement therapy. $\mathcal{J}$ Pediatr 1975;86:360-3.

3 Weichsel ME Jr. Thyroid hormone replacement therapy in the perinatal period: neurologic considerations. $\mathcal{J}$ Pediatr 1978;90:1035-8

4 Frost GJ, Parkin JM. Management of patients with congenital hypothyroidism. Br Med $\mathcal{F}$ 1985;290:1485-9.

5 Rezvani I, Di George AH. Reassessment of the daily dose of oral thyroxine for replacement therapy in hypothyroid children. $\mathcal{F}$ Pediatr 1977;90:291-7.

6 Paulus JW, Ranquin R, Parizel G. Systolic time intervals: a valuable parameter of thyroid function. Angiology 1980;31: $100-8$.

7 Plotnick GD, Vassar DL, Parisi AF, Hamilton BP, Carliner $\mathrm{NH}$, Fisher HL. Systolic time intervals in hypothyroidism; end organ function as a reflection of clinical status. Am f Med Sci 1978;277:263-8.

8 Greulich WW, Pyle SI. Radiographic atlas of skeletal development of hand and wrist. Stanford: Stanford University Press, 1959.

9 O'Malley BP, Abbott RJ, Barnett DB, Northover BJ, Rosenthal FD. Propranolol versus carbimazole as the sole treatment for thyrotoxicosis: A consideration of circulating thyroid hormone levels and tissue thyroid function. Clin Endocrinol 1982;16:545-52.

10 Gilman AG, Murad F. Thyroid and antithyroid drugs. In: Goodman M, Gilman AG, eds. The pharmacological basis of therapeutics 5th ed. New York: Macmillan, 1975:1403.

11 Fish LH, Schwartz HL, Cavanaugh J, Steffes MW, Bantle JP, Oppenheimer JH. Replacement dose, metabolism and bioavailability of levothyroxine in the treatment of hypothyroidism. $N$ Engl f Med 1987;316:764-70.

12 Sato T, Suzuki Y, Taketani T. Age related change in pituitary threshold for TSH release during thyroxine replacement therapy for cretinism. F Clin Endocrinol 1977;44: 553-8.

13 Schultz RM, Glassman MS, MacGillivray MH. Elevated threshold for thyrotrophin suppression in congenital hypothyroidism. Am $\mathcal{F}$ Dis Child 1980;134:19-20. 